SMAD, Rev. Eletrônica Saúde Mental Álcool Drog.

2021 jan.- mar.;17(1):32-38

DOI: 10.11606/issn. 1806-6976.smad.2021.156968

www.revistas.usp.br/smad/

Artigo Original

\title{
Nutrizes usuárias de drogas e o desfecho da amamentação: estudo de coorte*
}

\section{Silmara de Fátima Teixeira Ribeiro \\ (D) https://orcid.org/0000-0001-5932-3048 \\ Rosa Aurea Quintella Fernandes ${ }^{1}$ \\ (D) https://orcid.org/0000-0003-1037-7840}

*Artigo extraído de dissertação de mestrado "Aleitamento materno em mulheres usuárias de drogas ilícitas", apresentada à Universidade Guarulhos, Guarulhos, SP, Brasil.

Universidade Guarulhos, Guarulhos, SP, Brasil.
Objetivo: conhecer o desfecho do aleitamento materno de mães usuárias de drogas lícitas e ilícitas, verificar a gravidade da dependência e relacionar com o tempo de aleitamento. Método: estudo de coorte com 108 mulheres. Utilizou-se o Alcohol Smoking and Substance Involvement Screening Test para estabelecer a dependência a drogas. A manutenção da amamentação foi avaliada no $7^{\circ}, 15^{\circ}$ e $30^{\circ}$ dias de nascimento. Para avaliar a continuidade do aleitamento materno, utilizou-se análise de sobrevivência de Kaplan-Meier e de Cox. Resultados: a maconha teve escore de uso abusivo para $42,6 \%$ das mães e a cocaína para 37\%. A média de aleitamento foi de 28,8 dias. O uso abusivo de tabaco e álcool representou, respectivamente, 6,6 e 11 vezes mais risco de descontinuar a amamentação. Não foi possível estabelecer a mesma relação para maconha e cocaína. Conclusão: as mães usuárias de drogas praticaram o aleitamento exclusivo, mesmo aquelas que consomem maconha e cocaína de modo abusivo. O consumo de drogas, mesmo lícitas, representa um risco para o desmame precoce.

Descritores: Aleitamento Materno; Drogas Ilícitas; Enfermagem Materno-Infantil; Cuidados de Enfermagem; Usuários de Drogas.

\section{Como citar este artigo}

Ribeiro SFT, Fernandes RAQ. Drug-using nursing mothers and breastfeeding outcome: a cohort study. SMAD, Rev Eletrônica Saúde Mental Álcool Drog. 2021 jan.- mar.;17(1):32-38. doi: https://dx.doi.org/10.11606/issn.1806-6976.smad.2021.156968 


\title{
Nursing mothers that use drugs and the breastfeeding outcome: a cohort study
}

\begin{abstract}
Objective: to identify the breastfeeding outcome for mothers that use licit and illicit drugs, to verify the severity of their dependence and to relate this to the breastfeeding duration. Method: cohort study with 108 women. The Alcohol Smoking and Substance Involvement Screening Test (ASSIST) was used to establish drug dependence. Breastfeeding maintenance was assessed on the $7^{\text {th }}, 15^{\text {th }}$ and $30^{\text {th }}$ days of life. To assess the breastfeeding continuity, Kaplan-Meier and Cox survival analysis was used. Results: $42.6 \%$ of the mothers presented an score indicative of abuse for marijuana and $37.0 \%$ for cocaine. The mean length of breastfeeding was 28.8 days. The abuse of tobacco and alcohol represented, respectively, 6.6 and 11.0 times more risk of discontinuing the breastfeeding. It was not possible to establish this relationship in relation to marijuana and cocaine. Conclusion: the mothers that used drugs practiced exclusive breastfeeding, even those that used marijuana and cocaine in an abusive way. Drug use, even if legal, represents a risk for early weaning.
\end{abstract}

Descriptors: Breast Feeding; Street Drugs; Maternal-Child Nursing; Nursing Care; Drug Users.

\section{Nutrices consumidoras de drogas y resultado de la lactancia materna: un estudio de cohorte}

Objetivo: conocer el resultado de la lactancia materna para las madres que consumen drogas lícitas e ilícitas, verificar la gravedad de la dependencia y relacionarla con la duración de la lactancia materna. Métodos: estudio de cohorte con 108 mujeres. Se utilizó el Alcohol Smoking and Substance Involvement Screening Test para establecer la adicción a las drogas. El mantenimiento de la lactancia materna se evaluó a los 7, 15 y 30 días de vida. Para evaluar la continuidad de la lactancia materna se utilizó el análisis de supervivencia de Kaplan-Meier y Cox. Resultados: la marihuana tuvo una puntuación de uso abusivo para el $42,6 \%$ de las madres y la cocaína el 37\%. El promedio de lactancia fue de 28,8 días. El consumo abusivo de tabaco y alcohol representaba, respectivamente, 6,6 y 11 veces más riesgo de interrumpir la lactancia materna. No fue posible establecer la misma relación para la marihuana y la cocaína. Conclusión: las madres que consumen drogas han practicado la lactancia materna exclusiva, incluso las que abusan de la marihuana y la cocaína. El consumo de drogas, incluso las lícitas, representa un riesgo para el destete precoz.

Descriptores: Lactancia Materna; Drogas Ilícitas; Enfermería Maternoinfantil; Atención de Enfermería; Consumidores de Drogas. 


\section{Introdução}

O Aleitamento Materno Exclusivo (AME) é recomendado do nascimento até o $6^{\circ}$ mês de vida, por ser considerado a nutrição adequada ao crescimento saudável da criança e por reduzir a morbimortalidade infantil ${ }^{(1)}$.

O Aleitamento Materno (AM), principalmente o exclusivo, é fundamental para a diminuição das taxas de morbimortalidade infantil, sendo este um dos motivos pelos quais tem sido estimulado, apoiado e promovido por instituições nacionais e internacionais.

Entretanto, a indicação do AM para crianças de mães usuárias de drogas ilícitas não é consenso entre os autores. Há falta de uniformidade na opinião de especialistas, assim como de consenso sobre essa matéria(2-3). A divergência de opiniões, inclusive das entidades públicas responsáveis pelas diretrizes e normas de saúde, dificulta a atuação dos profissionais que se deparam com múltiplos desafios na orientação dessas mães(3-4).

O Ministério da Saúde (MS) enfatiza, em suas diretrizes sobre amamentação, a divergência de opinião entre a Sociedade Americana de Pediatria, que contraindica o uso de substâncias psicoativas na lactação, e a posição da Organização Mundial de Saúde (OMS), que não considera contraindicada a amamentação e recomenda que as nutrizes não façam uso dessas substãncias ${ }^{(5)}$.

A Sociedade Brasileira de Pediatria, por sua vez, não é favorável a que mães usuárias de drogas de abuso amamentem seus filhos. Orienta que mães que consomem drogas ocasionalmente devem suspender o aleitamento por tempo que varia de acordo com a droga consumida(6).

Entretanto, mães usuárias, mesmo aquelas que têm desejo de amamentar, não têm controle de si para evitar o consumo desas substâncias ou mesmo para administrar o intervalo de tempo desejável entre o consumo e o aleitamento, o que coloca em risco os lactentes. Essa realidade e a falta de serviços de qualidade para apoio à mãe usuária dificultam a decisão do profissional na orientação sobre aleitamento materno(6).

Estudo reforça que há muita confusão na comunidade científica sobre esse tema e que há casos de efeitos clínicos de toxicidade em crianças aleitadas por mães dependentes, mas há poucos estudos que confirmem os riscos reais para a criança(7).

Por outro lado, não foram identificadas publicações que evidenciem o desfecho da amamentação por mães usuárias de drogas, o que justifica as questões desse estudo: Mães usuárias de drogas lícitas e ilícitas amamentam seus filhos? Há relação entre o grau de dependência da droga e o tempo de aleitamento materno?

Os objetivos deste estudo foram: conhecer o desfecho do aleitamento materno de mães usuárias de drogas lícitas e ilícitas, verificar a gravidade da dependência e relacionar com o tempo de amamentação.

\section{Método}

Estudo de coorte realizado em um hospital de médio porte, localizado na zona leste da cidade de São Paulo, que atende pelo Sistema Único de Saúde e com demanda importante de mães usuárias de drogas. Os dados foram coletados pela própria pesquisadora no segundo semestre de 2015.

Participaram deste estudo 108 puérperas. O cálculo da amostra foi realizado pelo Programa STATS, e considerou-se a média mensal de partos (296), erro de $5 \%$ e nível de confiança de $95 \%$.

Participaram do estudo 108 mulheres que atenderam aos seguintes critérios de inclusão: confirmar ser usuária de drogas ilícitas; estar no puerpério (de 24 a 48 horas pós-parto); ter condições de responder aos questionários; estar em aleitamento materno no momento da alta; aceitar participar do estudo por 30 dias após o nascimento do bebê.

Para a coleta dos dados, foram utilizados três instrumentos: um para caracterização sociodemográfica, obstétrica e hábitos de vida das mulheres e englobou as variáveis: estado civil, idade, escolaridade, etnia, religião, exerce atividade remunerada, gestações, partos, abortos, pré-natal, número de consultas e se usuária de bebidas alcoólicas e tabaco.

O segundo instrumento foi o Alcohol, Smoking and Substance Involvement Screening Test (ASSIST). Esse teste foi desenvolvido por diversos pesquisadores sob a coordenação da Organização Mundial de Saúde (OMS) e tem como objetivo detectar o uso de álcool, tabaco e outras substâncias psicoativas. $O$ instrumento foi traduzido para várias línguas, inclusive para o português falado no Brasil, e foi testado quanto à sua confiabilidade e facilidade de aplicação( ${ }^{(8)}$. As propriedades psicométricas da versão brasileira do ASSIST se mostraram satisfatórias, o que recomenda a sua aplicação em pacientes de serviços de atenção primária e secundária.

O instrumento está estruturado em oito questões sobre o uso de nove classes de substâncias psicoativas (tabaco, álcool, maconha, cocaína, estimulantes, sedativos, inalantes, alucinógenos e opioides). Cada resposta corresponde a um escore que varia de zero a quatro ( 0 a 4 ), sendo que a soma total dos escores pode variar de zero a vinte ( 0 a 20). De acordo com o escore obtido, o usuário recebe uma classificação de risco de zero a três ( 0 a 3 ) indicando uso ocasional, de quatro a quinze ( 4 a 15 ) abuso e escore acima de 16 é sugestivo de dependência( ${ }^{(8)}$.

O terceiro instrumento foi aplicado apenas nos encontros, para monitorar a prática do AM pelas mães $\left(7^{\circ}\right.$, $15^{\circ}$ e $30^{\circ}$ dias) e constou das variáveis: tipo de aleitamento praticado e utilização ou não de drogas no período de amamentação e espaço para observações do pesquisador.

Os dados foram coletados em duas etapas. $\mathrm{Na}$ primeira, as mães foram abordadas no puerpério, entrevistadas individualmente em local privativo e 
aplicados os instrumentos de caracterização da amostra e o ASSIST. A segunda etapa foi o monitoramento que ocorreu nos $7^{\circ}, 15^{\circ}$ e $30^{\circ}$ dias após o parto, as mães foram entrevistadas nos retornos agendados, para esses períodos, no ambulatório de aleitamento materno da instituição, ocasião em que eram questionadas sobre o uso de drogas ilícitas e o tipo de aleitamento praticado até aquele momento. Além disso, havia um espaço para anotações do pesquisador sobre suas observações.

Para avaliar a continuidade do aleitamento materno, foram utilizados modelos de análise de sobrevivência de Kaplan-Meier e de Cox (modelo semiparamétrico). Para todos os testes estatísticos, foi adotado nível de significância de $5 \%(p<0,05)$. As análises estatísticas foram realizadas utilizando-se SPSS 20.0 e Stata 12 .

Foram seguidos os princípios éticos para pesquisa envolvendo seres humanos da Resolução 466/12 do Conselho Nacional de Saúde no Brasil. O estudo foi aprovado sob nº 665.183 .

\section{Resultados}

O perfil sociodemográfico das mães aponta idade média de 23,7 (DP 6,0), faixa etária predominante entre 20 e 29 anos (62\%). A maioria com companheiro (51,9\%), branca $(52,9 \%)$ e sem atividade remunerada $(75,9 \%)$. Nível de escolaridade baixo com predomínio de fundamental incompleto (33,3\%) e 41,7\% professando a religião católica.

Os dados obstétricos evidenciaram que $67,6 \%$ nunca tiveram aborto, $87 \%$ fizeram pré-natal e que $42,6 \%$ eram primíparas. Aquelas que realizaram o acompanhamento pré-natal, 94 (64,9\%), compareceram a cinco (5) consultas ou mais. Entretanto,13\% não realizaram nenhuma consulta. Quanto aos hábitos de vida, a maioria era tabagista $(58,3 \%)$ e não se considerava etilista $(57,4 \%)$.

A classificação de risco das mães realizada no puerpério, para o uso de drogas lícitas e ilícitas pela aplicação do ASSIST, está demonstrada na Tabela 1.

Tabela 1 - Distribuição do número e percentual de mães, segundo as categorias do escore ASSIST* para o uso de substâncias lícitas e ilícitas, Guarulhos, SP, Brasil, 2015 $(n=108)$

\begin{tabular}{llcc}
\hline Variável & Categoria (pontuação) & $\mathbf{n}$ & (\%) \\
\hline Tabaco & Uso ocasional (0 a 3) & 98 & 90,7 \\
& Sugestivo de abuso (4 a 15) & 10 & 9,3 \\
Álcool & Uso ocasional (0 a 3) & 101 & 93,5 \\
& Sugestivo de abuso (4 a 15) & 06 & 5,6 \\
\hline \multirow{2}{*}{ Maconha } & Sugestivo de dependência & 01 & 0,9 \\
& (16 a 20) & 61 & 56,5 \\
& Uso ocasional (0 a 3) & 46 & 42,6 \\
& Sugestivo de abuso (4 a 15) & & (continua...)
\end{tabular}

\begin{tabular}{|c|c|c|c|}
\hline Variável & Categoria (pontuação) & $\mathbf{n}$ & $(\%)$ \\
\hline & $\begin{array}{l}\text { Sugestivo de dependência } \\
(16 \text { a } 20)\end{array}$ & 01 & 0,9 \\
\hline \multirow[t]{3}{*}{ Cocaína } & Uso ocasional (0 a 3) & 63 & 58,4 \\
\hline & Sugestivo de abuso (4 a 15) & 40 & 37,0 \\
\hline & $\begin{array}{l}\text { Sugestivo de dependência } \\
(16 \text { a } 20)\end{array}$ & 05 & 4,6 \\
\hline Anfetaminas & Uso ocasional (0 a 3) & 108 & 100,0 \\
\hline \multirow[t]{2}{*}{ Inalantes } & Uso ocasional (0 a 3) & 107 & 99,1 \\
\hline & Sugestivo de abuso (4 a 15) & 01 & 0,9 \\
\hline \multirow[t]{2}{*}{ Sedativos } & Uso ocasional (0 a 3) & 107 & 99,1 \\
\hline & Sugestivo de abuso (4 a 15) & 01 & 0,9 \\
\hline Alucinógeno & Uso ocasional (0 a 3) & 108 & 100,0 \\
\hline Opiáceos & Uso ocasional (0 a 3) & 108 & 100,0 \\
\hline Total & & 108 & 100,0 \\
\hline
\end{tabular}

${ }^{*}$ ASSIST $=$ Alcohol, Smoking and Substance Involvement Screening Test

Evidenciou-se que a maioria das mães teve escores compatíveis com o uso ocasional para todos os tipos de drogas analisadas. Escores sugestivos de abuso foram identificados com maiores percentuais para maconha ( $n=46 ; 42,6 \%)$ e cocaína ( $n=40 ; 37 \%)$.

Em relação ao aleitamento materno, das 108 mães que compuseram a amostra, $86(79,6 \%)$ compareceram ao retorno do $70 \mathrm{dia}, 75(69,4 \%)$ ao do $15^{\circ}$ dia e 57 $(52,7 \%)$ ao do $30^{\circ}$ dia. O tempo médio de continuidade do aleitamento materno exclusivo foi de 28,8 dias $($ IC95\% $=[27,8 ; 29,9])$.

No momento da alta hospitalar, as 108 mães (100\%) que participaram do estudo estavam aleitando exclusivamente. No primeiro retorno, 22 mães não compareceram à consulta, ou seja, houve um missing (perda) de 20,4\%. Daquelas que retornaram, 55 (64\%) estavam em AME. A maioria das mães que retornaram no $15^{\circ}$ e no $30^{\circ}$ dia manteve o $\operatorname{AME}(57,3 \%$ e $52,7 \%$, respectivamente). Entretanto, o missing aumentou com a continuidade do monitoramento $\left(30,6 \%\right.$ no $15^{\circ}$ dia e $47,3 \%$ no $30^{\circ}$ ).

Dentre as mães que foram acompanhadas até o $30^{\circ}$ dia (57), observaram-se nove (9) casos de descontinuidade de aleitamento materno (15,8\%). Todas as mães que descontinuaram o aleitamento, em todos os períodos analisados, faziam uso das quatro substâncias (tabaco, álcool, maconha e cocaína (Tabela 2).

Tabela 2 - Resultados do modelo de regressão de Cox multivariada, Guarulhos, SP, Brasil, $2015(n=108)$

\begin{tabular}{lcc}
\hline ASSIST $^{*}$ & $\begin{array}{c}\text { Razão de Riscos } \\
(\text { IC-95\%) }\end{array}$ & p-value \\
\hline Tabaco & & \\
Não faz uso (referência) & 1,00 & - \\
Uso ocasional (1 a 3) & $0,00^{\ddagger}$ & 0,99
\end{tabular}




$\begin{array}{lcc}\text { Sugestivo de abuso (4 a 15) } & 6,64(1,59-27,83) & 0,01 \\ \text { Álcool } & & - \\ \text { Não faz uso (referência) } & 1,00 & 0,99 \\ \text { Uso ocasional (1 a 3) } & 0,00^{\ddagger} & 0,99 \\ \text { Sugestivo de abuso (4 a 15) } & 0,00^{\ddagger} & 0,02 \\ \text { Sugestivo de dependência (16 a 22) } & 11,01(1,28-94,68) & 0\end{array}$

${ }^{*}$ ASSIST $=$ Alcohol, Smoking and Substance Involvement Screening Test;

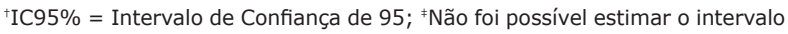
de confiança de $95 \%$ por falta de precisão

O modelo de regressão de Cox apresentado (Tabela 2) tem como variáveis preditoras as classificações do ASSIST, para tabaco e álcool, das mães de bebês que compareceram às consultas (significantes a $10 \%$ na análise univariada). No modelo de Cox, a função de sobrevivência é expressa em função do risco (hazard function) e a interpretação do modelo é realizada na forma de razão das funções de riscos.

Permaneceu significante (Tabela 2), no modelo final, a classificação do ASSIST para tabaco - sugestivo de abuso $(p=0,01)$ - e para álcool - sugestivo de dependência $(p=0,02)$. Verificou-se que as mães com uso sugestivo de abuso para tabaco têm 6,6 vezes mais risco de descontinuar o aleitamento materno do que aquelas que não fazem uso de tabaco, e as mães com uso sugestivo de dependência de álcool têm 11,0 vezes mais risco de descontinuar o aleitamento materno do que aquelas que não fazem uso de álcool. Não foi possível estabelecer o risco para maconha e cocaína nos períodos analisados, pois não houve descontinuidade do aleitamento.

\section{Discussão}

Estudos relativos ao consumo de drogas e sua relação com a continuidade do aleitamento materno não foram encontrados, o que dificulta comparações com os dados da pesquisa em questão.

A maioria das mulheres (87\%) fez pré-natal, o que representa uma oportunidade para a detecção do consumo de drogas, orientação e encaminhamento a serviço de apoio ao usuário, quando necessário. Os profissionais que prestam assistência às mulheres no período gravídico puerperal necessitam estar alertas a sinais de seu envolvimento com drogas psicoativas, para garantir a intervenção adequada.

O Colégio Americano de Ginecologia e Obstetrícia recomenda que todas as mulheres sejam rastreadas, para o uso de drogas ilícitas, álcool e tabaco, durante o prénatal(9). Entretanto, nem todos os profissionais conhecem os instrumentos de detecção de drogas ilícitas ou estão habilitados para realizar intervenções com esse tipo de clientela, o que demandaria preparo para intervenções, mesmo as breves ${ }^{(10-11)}$.

Neste estudo, pelo resultado do ASSIST, as drogas apontadas com consumo sugestivo de abuso foram a maconha e a cocaína. Pesquisa qualitativa realizada com puérperas usuárias de drogas identificou que as mais usadas foram também a maconha, a cocaína/crack e que as mulheres tiveram dificuldade de ficar em abstinência, no período gestacional(12), o que provavelmente continuaria a ocorrer durante a lactação.

No estudo atual, durante o monitoramento, observou-se que todas as mães que descontinuaram o aleitamento em todos os períodos analisados faziam uso das quatro drogas (tabaco, álcool, maconha e cocaína).

Quanto ao tabaco e álcool, a maioria das mães as consumiam. Para alguns profissionais de saúde, esse consumo não é considerado relevante, e não é dada a devida importância para orientar as mulheres sobre seus riscos no período de lactação. Entretanto, nesse estudo, as mães com uso sugestivo de abuso para tabaco tiveram 6,6 vezes mais risco de descontinuar o aleitamento materno e as mães com uso sugestivo de dependência de álcool elevaram essa chance para 11 vezes.

Estudo que analisou as causas do desmame precoce identificou associação entre o consumo de bebida alcoólica, na gestação, à descontinuidade do aleitamento materno(13).

Pesquisa com 157 lactantes identificou que 12\% faziam uso de bebida alcoólica, mas todas foram consideradas de baixo risco para transtornos causados pelo consumo. Apesar de os autores considerarem esse percentual baixo, os lactentes foram expostos a risco com o hábito de suas mães ${ }^{(14)}$.

A Sociedade Brasileira de Pediatria, em sua classificação de risco de uso de substâncias durante a lactação, considera a nicotina como possivelmente compatível com a lactação, o álcool como possivelmente perigoso e outras drogas como maconha, cocaína, crack e metanfetaminas como perigosas, mas recomenda explicitamente que mães usuárias regulares de drogas de abuso não devem amamentar seus filhos. Para as usuárias ocasionais, sugere a suspensão do aleitamento por um tempo variável, de acordo com a droga consumida(6).

É fundamental que os profissionais que acompanham as mulheres no período gravídico puerperal estejam seguros das orientações e recomendações das agências de saúde sobre o aleitamento materno e consumo de substâncias lícitas e ilícitas, de modo a garantir uma assistência resolutiva.

O monitoramento da continuidade do aleitamento das mães que compuseram a amostra identificou um tempo médio de aleitamento materno exclusivo de 28,8 dias. Considerando que o acompanhamento se deu até o $30^{\circ} \mathrm{dia}$, o resultado é importante, tendo em vista que a pesquisa nacional sobre AM de crianças de 0 a 6 meses identificou mediana de 54,1 dias de $\mathrm{AME}^{(15)}$. O ideal para avaliar o desfecho do AME seria manter o monitoramento até os 6 meses da criança.

Por outro lado, percebeu-se, durante as consultas de acompanhamento, que as mães eram arredias, referiam sentir que eram tratadas pelos profissionais de modo 
diferente do das outras mães, o que talvez justificasse o missing (perdas) na continuidade do monitoramento. Esse sentimento é apontado por estudo que afirma que as usuárias de drogas são muito discriminadas ${ }^{(16)}$.

Considerando que as mães usuárias regulares de drogas amamentam seus filhos, como demonstrado na pesquisa, há que se ponderar sobre a importância de garantir suporte para essas mulheres na proteção de sua saúde e de seus bebês. Contudo, a baixa qualidade dos serviços aos usuários na atenção à saúde, em nosso meio, não garante a abstinência necessária para manter o aleitamento(6).

Os resultados do presente estudo não possibilitam realizar a associação do tempo de manutenção do AM com o uso de maconha e cocaína, mas demonstra chances de as mães desistirem de amamentar seus filhos quando consomem tabaco e álcool. Assim, com base nesses dados e na literatura(17), pode-se inferir que as mães com drogadição mantêm por menos tempo o AM.

Estudo realizado na Austrália que analisou resultados de crianças expostas à anfetamina durante a gestação identificou que as mães que consumiam drogas foram significativamente menos suscetíveis a amamentar do que as que não consumiam drogas $(27,0 \%$ e $41,6 \%$, respectivamente)(17).

Algumas publicações ${ }^{(4,7,18)}$ afirmam não haver evidências suficientes sobre a detecção da cocaína e da maconha no leite materno para recomendar com segurança a suspensão do AM, embora haja demonstração de que, mesmo em pequena porcentagem, o leite materno pode conter a droga consumida pela mãe e, dessa forma, expor a criança a tais substâncias ${ }^{(7,16,18)}$.

Estudo de revisão sobre mães que consomem crack/ cocaína enfocou que a amamentação e o cuidado dos recém-nascidos de nutrizes dependentes dessas drogas são aspectos que devem ser conduzidos com muito cuidado. $\mathrm{O}$ artigo destaca ainda que o Hospital das Clínicas de Porto Alegre não recomenda o AM para essas mães ${ }^{(19)}$.

Essa orientação difere da veiculada no hospital onde o estudo foi realizado, que não tinha, em seu protocolo, essa proibição, ou seja, as mães, mesmo consumindo drogas, eram incentivadas a amamentar, orientadas quanto aos riscos e encaminhadas a serviço para acompanhamento de dependentes químicos.

Essas mães eram orientadas também a retornarem ao ambulatório de aleitamento, mas não havia atendimento especial direcionado a elas por conta de sua condição e nem controle da continuidade do consumo de drogas, do AM e da adesão ao tratamento.

Dentre as limitações deste estudo, ressalta-se o número limitado de mães que continuou a comparecer às consultas de retorno até o final $(52,8 \%)$, o que reduziu muito a amostra para o monitoramento proposto.
Essa clientela, de modo geral, tem dificuldade em dar continuidade às atividades de saúde.

\section{Conclusão}

As mães usuárias de drogas lícitas ou ilícitas amamentam seus filhos, e a maioria daquelas que continuou o acompanhamento após o parto manteve o AME. O Teste ASSIST evidenciou para a maioria das mulheres escores compatíveis com uso ocasional para todas as drogas analisadas e uso sugestivo de abuso para maconha e cocaína. O uso de tabaco e álcool são fatores de risco para o desmame precoce. Não foi possível estabelecer essa relação para a maconha e a cocaína, devido ao tempo de acompanhamento das mulheres.

\section{Referências}

1. Ministério da Saúde. Secretaria de Atenção à Saúde. Departamento de Atenção Básica (BR). Saúde da criança: aleitamento materno e alimentação complementar. [Internet]. 2a ed. Brasília: Ministério da Saúde; 2015. [Acesso 16 out 2018]. Disponível em: http://bvsms. saude.gov.br/bvs/publicacoes/saude_crianca_aleitamento_ materno_cab23.pdf

2. Eidelman AI. Drug use by the breastfeeding mother: A medical and societal challenge. Breastfeed Med. [Internet]. 2015 [cited 2018 Oct 16]; 10(3):133-4. Available from: https://www.liebertpub.com/doi/abs/10.1089/ bfm.2015.9993?journalCode $=$ bfm

3. Reece-Stremtan SR, Marinelli KA. Guidelines for breastfeeding and substance use or substance use disorder, revised 2015. Breastfeed Med. [Internet]. 2015 [cited 2018 Oct 16]; 10(3):135-41. Available from: https://www.ncbi. nlm.nih.gov/pmc/articles/PMC4378642/

4. D'Apolito K. Breastfeeding and substance abuse. Clin Obstet Gynecol. [Internet]. 2013 [cited 2018 Oct 16]; 56(1):202-11. Available from: https://insights.ovid.com/ pubmed?pmid $=23337847$

5. Ministério da Saúde. Secretaria da Atenção à Saúde. Departamento de Ações Programáticas e Estratégicas (BR). Amamentação e uso de medicamentos e outras substâncias. 2a ed. [Internet]. Brasília: Ministério da Saúde; 2010. [Acesso 16 out 2018] Disponível em: http:// bvsms.saude.gov.br/bvs/publicacoes/amamentacao_uso_ medicamentos_2ed.pdf

6. Sociedade Brasileira de Pediatria. Departamento Científico de Aleitamento Materno. O uso de medicamentos e outras substâncias pela mulher durante a amamentação. [Internet]. 2017. [Acesso 16 out 2018] Disponível em: http://www.sbp.com.br/fileadmin/user_upload/ Aleitamento_-_Uso_Medicam_durante_Amament.pdf 7. Fríguls $B$, Joya $X$, García-Algar $O$, Pallás $C R$, Vall O, Pichini $\mathrm{S}$. A comprehensive review of assay methods to determine drugs in breast milk and the safety of breastfeeding when 
taking drugs. Anal Bioanal Chem. [Internet]. 2010. [cited 2018 Oct 16]; 397(3):1157-79. Available from: https:// link.springer.com/article/10.1007\%2Fs00216-010-3681-0 8. Henrique IF, Micheli D, Lacerda RB, Lacerda LA, Formigoni ML. Validation of the Brazilian version of Alcohol, Smoking and Substance Involvement Screening Test (ASSIST). Rev Assoc Med Bras. [Internet]. 2004 [cited 2018 Oct 16]; 50(2):199-206. Available from: http:// www.scielo.br/scielo.php?script=sci_arttext\&pid=S010442302004000200039\&Ing=en

9. Krans EE, Cochran G, Bogen DL. Caring for opioid dependent women: prenatal and postpartum care considerations. Clin Obstet Gynecol. [Internet]. 2015; [cited 2018 Oct 16];.58(2):370-9. Available from: https:// www.ncbi.nlm.nih.gov/pmc/articles/PMC4607033

10. Silva AC, Lucchese R, Vargas LS, Benício PR, Vera I. Application of the Alcohol, Smoking and Substance Involvement Screening Test (ASSIST) instrument: an integrative review. Rev Gaúcha Enferm. [Internet]. 2016 [Acesso 16 out 2018]; 37(1):e52918. Disponível em: http://www.scielo.br/scielo.php?script=sci_ arttext\&pid $=$ S1983-14472016000100501\&lng=pt\&tlng= 11. Coutinho T, Coutinho CM, Coutinho LM. Prenatal care of illicit drugs users. Femina. [Internet]. 2014 [cited 2018 Oct 16]; 42(1):11-8. Available from: http://files.bvs.br/ upload/S/0100-7254/2014/v42n1/a4808.pdf

12. Portela GLC, Barros LM, Frota NM, Landim APP, Caetano JÁ, Farias FLR. Perception of pregnant on consumption of illicit drugs in pregnancy. SMAD. Rev Eletrônica Saúde Mental Álcool Drog. [Internet] 2013. [cited 2018 Oct 16]; 9(2):58-63. Available from: http://pepsic.bvsalud.org/scielo.php?script $=$ sci_ arttext\&pid=S1806-69762013000200002\&Ing=pt\&nrm =iso\&tlng=en

13. Sanches MTC, Buccini GS, Gimeno SGA, Rosa TEC, Bonamigo AW. Factors associated with interruption of exclusive breastfeeding in low birth weight infants receiving primary care. Cad Saúde Pública [Internet]. 2011 [cited 2018 out. 16]; 27(5):53-965. Available from: http://www. scielo.br/scielo.php?script=sci_arttext\&pid $=$ S0102-311X20 $11000500013 \&$ Ing $=p t \&$ tIng $=p t$

14. Nascimento ALV, Souza AFO, Amorim ACR, Leitão MBS, Maio R, Burgos MGPA. Alcohol intake in lactating women assisted in a University Hospital. Rev Paul Pediatr. [Internet]. 2013 June [cited 2018 Oct 16]; 31(2):198-204. Available from: http://www.scielo.br/scielo.php?script=sci_ arttext\&pid=S0103-05822013000200010\&Ing=en
15. Venancio SI, Escuder MML, Saldiva SRDM, Giugliani ERJ. Breastfeeding practice in the Brazilian capital cities and the Federal District: ] Pediatr. [Internet]. 2010 [cited 2018 Oct 16]; 86(4):317-24. Available from: http:// www.scielo.br/scielo.php?script=sci_arttext\&pid=S002175572010000400012\&lng=en

16. Botelho APM, Rocha RC, Melo VH. Cocaine/crack use and dependence in pregnancy, delivery and puerperium. Femina. [Internet]. 2013 [cited 2018 Oct 16]; (4)1:23-32. Available from: http://files.bvs.br/ upload/S/0100-7254/2013/v41n1/a3777.pdf

17. Oei J, Abdel-Latif ME, Clark R, Craig F, Lui K. Shortterm outcomes of mothers and infants exposed to antenatal amphetamine. Arch Dis Child fetal Neonatal Ed. [Internet].2010 [cited 2018 Oct 16]; 95(1):36-41. Available from: https://fn.bmj.com/content/95/1/F36.long 18. American College of Obstetricians and Gynecologists Committee on Obstetric Practice. Marijuana use during pregnancy and lactation. Obstet Gynecol. [Internet]. 2017 [cited 2018 Oct 18]; 130(4):e205-9. Available from: https://www.acog.org/-/media/Committee-Opinions/ Committee-on-Obstetric-Practice/co722.pdf?dmc $=1 \&$ ts $=20181018 \mathrm{~T} 1320024672$

19. Martins-Costa SH, Vettorazzi J, Cecin GKG, Maluf JMRA, Stumpf CC, Ramos JGL. Crack: the new obstetric epidemic. Rev HCPA. [Internet]. 2013 [cited 2018 Oct 18]; 33(1):55-64. Available from: https://seer.ufrgs.br/hcpa/ article/viewFile/33833/25672

\section{Contribuição dos Autores}

Concepção e planejamento do estudo: Silmara Fatima Teixeira Ribeiro, Rosa Aurea Quintella Fernandes. Obtenção dos dados: Silmara Fatima Teixeira Ribeiro. Análise e interpretação dos dados: Silmara Fatima Teixeira Ribeiro, Rosa Aurea Quintella Fernandes.

Análise estatística: Rosa Aurea Quintella Fernandes. Redação do manuscrito: Silmara Fatima Teixeira Ribeiro, Rosa Aurea Quintella Fernandes. Revisão crítica do manuscrito: Rosa Aurea Quintella Fernandes.

Todos os autores aprovaram a versão final do texto. Conflito de interesse: os autores declararam que não há conflito de interesse.
Copyright $\odot 2021$ SMAD, Rev. Eletrônica Saúde Mental Álcool Drog. Este é um artigo de acesso aberto distribuído sob os termos da Licença Creative Commons CC BY-NC.

Esta licença permite que outros remixem, adaptem e criem a partir do seu trabalho para fins não comerciais, e embora os novos trabalhos tenham de Ihe atribuir o devido crédito e não possam ser usados para fins comerciais, os usuários não têm de licenciar esses trabalhos derivados sob os mesmos termos. 